\title{
Longitudinal Gradient-coil with Improved Uniformity within the Volume of Interest
}

\author{
Gabriela A. Domínguez, Agustín Romero, Esteban Anoardo \\ Laboratorio de Relaxometría y Técnicas Especiales (LaRTE), FaMAF - Universidad Nacional de Córdoba e IFEG- \\ CONICET, Córdoba-Argentina
}

\begin{abstract}
We present a longitudinal magnetic field gradient-coil with optimized uniformity within the volume of interest (VOI). The accuracy of the optimization algorithm was confronted with measurements of the spatial dependence of the magnetic field within the VOI in a prototype-coil. The proposed device integrates the gradient-unit of a fast-fieldcycling magnetic resonance imaging (MRI) apparatus of own design.
\end{abstract}

Resumen-Se presenta una bobina de gradiente de campo magnético longitudinal con uniformidad optimizada dentro del volumen de interés (VOI). La precisión del algoritmo de optimización es cotejada con mediciones de la dependencia espacial del campo magnético en un prototipo. Este dispositivo integra la unidad de gradientes de un aparato de imágenes por resonancia magnética (IRM) con ciclado rápido de campo de diseño propio.

\section{INTRODUCTION}

Since the pioneering work of Sir Peter Mansfield and Paul C. Lauterbur [1]-[2], MRI has been extensively used in biomedical diagnosis and research, as well as in other areas like material science and security. A key feature to build an image using nuclear magnetic resonance (NMR) is the addition of magnetic field gradients (in superposition to the main magnetic field) to encode the space. Since the quality of the image strongly depends on the magnetic field gradients, huge effort has been made to improve this point, at both basic research and engineering. As a consequence, a plenty of patents and scientific papers have been published on this topic in the last 30 years.

On the other side, in the fast-field-cycling (FFC) NMR technique, the main field is cycled both in intensity and time in order to allow low-field experiments with a reasonable signal-to-noise ratio [3]-[5]. A clear advantage of the combined scheme FFC-MRI resides in the enhancement of $\mathrm{T}_{1}$-weigthed (spin-lattice relaxation time) contrasts at low fields. This essential feature is a consequence of enhanced differences between the relaxation times associated to the diverse components of the sample [6]-[8]. Relaxationweighted images obtained by this technique are otherwise impossible [9]-[10].

In this work we present the design of a longitudinal gradient-coil whose gradient uniformity within the VOI is optimized in terms of the coil dimensions. In FFC technology it is usually mandatory to minimize the magnet bore to fit within the minimum possible space according to the sample volume and probe space requirements (which in turn must also be designed attending this restriction). This is a consequence of the critical loss of magnetic field intensity in this kind of magnets as the bore is increased, i.e., the more compact the magnet the stronger the magnetic field for a given power. In our case, the need of gradient-coils inserted within the magnet bore, and radially between the magnet and the radio-frequency (RF) probe (see figure 1), requires a careful and efficient design of both the gradient and RF units. Here we focus in the optimization of a longitudinal gradient-coil (cylindrical geometry) with maximal uniformity in the radial direction. In particular, we deal with the maximum radial uniformity that can be achieved for a fixed coil-diameter, while also keeping an eye on the uniformity along the longitudinal dimension.

\section{MATHEMATICAL MODEL}

In mathematical terms the problem consists in finding the optimal distribution of turns of wire wound on a cylindrical body of $51 \mathrm{~mm}$ of diameter that will produce a linear gradient within the cylindrical VOI formed by the revolution of a centered plane of $30 \times 50 \mathrm{~mm}$, with a maximum uniformity deviation of $5 \%$. Since the thermomechanical stress of the winding may affect the gradient uniformity, and considering the switchable nature of the coil $^{1}$, the solution should also consider the resistance and inductance of the coil.

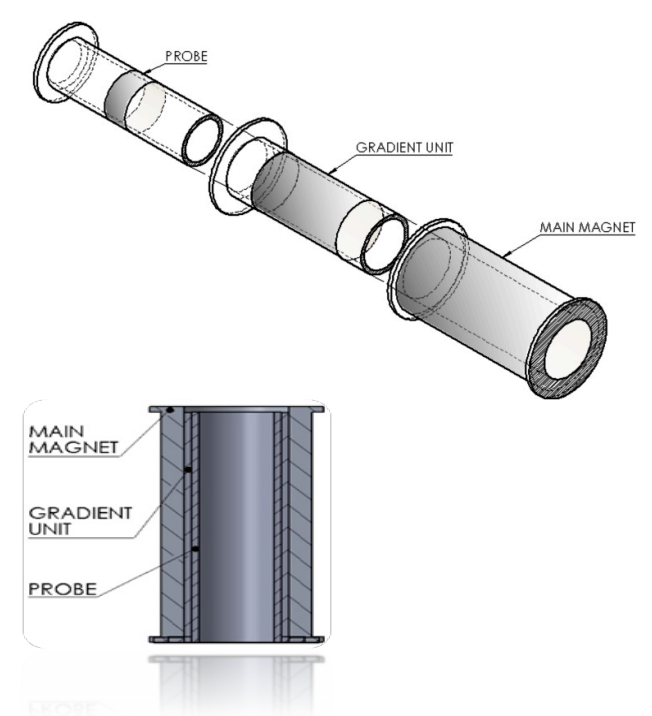

Fig 1. Schematic representation of the FFC-MRI assembly: magnet, gradient unit and RF probe. Shadowed surfaces indicate the active zones. The inner diameter of the probe is $32 \mathrm{~mm}$ and the outer diameter is $40 \mathrm{~mm}$. For the gradient unit (tree coils): inner diameter $47 \mathrm{~mm}$, outer $63 \mathrm{~mm}$. And for the main magnet the internal diameter is $65 \mathrm{~mm}$.

\footnotetext{
${ }^{1}$ The switching performance of the coil may also strongly depend on the gradient amplifier. "Slow" coils can be partly compensated with proper electric networks and amplifier technology.
} 


\section{A. Discretization}

Due to symmetry reasons, we reduced computational time by considering half of the cylindrical VOI. Since we are dealing with a gradient-coil, it is important to remark that the other half of the VOI corresponds to the mirror image of the calculated half, but with reverse current. The resulting calculated magnetic field map then corresponds to a plane whose revolution around the axis of the cylinder gives the solution in the volume (see figure 2).

Initially we consider an ideal coil set up by $\mathrm{N}$ equispaced non-pitched turns of wire (rings) along the z-component of a cylindrical surface. The current of each ring is independent from each other.

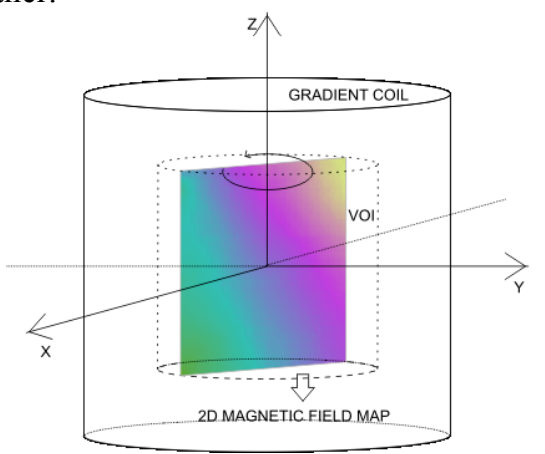

Fig 2. Geometry of the problem. In color: example of a bisector plane where the magnetic field-map is calculated. The revolution of the plane covers the VOI.

Based on the Biot-Savart, law we calculate the magnetic field generated by this set as [11]:

$$
\begin{gathered}
B_{z}\left(\vec{r}_{k}\right)=\sum_{n=1}^{N} I_{n} c_{n}\left(\vec{r}_{k}\right) \\
c_{n}\left(\vec{r}_{k}\right)=\frac{\mu_{0}}{4 \pi} \int\left[\frac{d \vec{l} \times \vec{r}}{r_{k}^{2}}\right] .
\end{gathered}
$$

Here, $I_{n}$ are the currents of the rings while $c_{n}\left(\vec{r}_{k}\right)$ stands for a geometrical factor.

Transversal components are negligible and completely irrelevant for the calculation. Therefore, we only consider the $z$-component of the magnetic field at $\vec{r}_{k}$ positions within the VOI and its surroundings. To find the optimal combination of currents that generates the required gradient (target), we introduce a cost function [12]:

$$
\Phi=\frac{1}{2} \sum_{k=1}^{K} \omega\left(\vec{r}_{k}\right)\left[B_{z}\left(\vec{r}_{k}\right)-B_{z}^{t}\left(\vec{r}_{k}\right)\right]^{2}+\frac{\alpha}{2} \sum_{n=1}^{N} R_{n} I_{n}^{2}
$$

where $\omega\left(\vec{r}_{k}\right)$ and $\alpha$ are weighting factors. This function minimizes the quadratic deviation of the field at the $K$ target points, and the dissipated power. The $I_{n}$ values which minimize $\Phi$ outcome from solving the $n \times n$ first-order simultaneous equations obtained from forcing all $\partial \Phi / \partial I_{n}$ to zero. Figure 3 shows the current density obtained after this process.

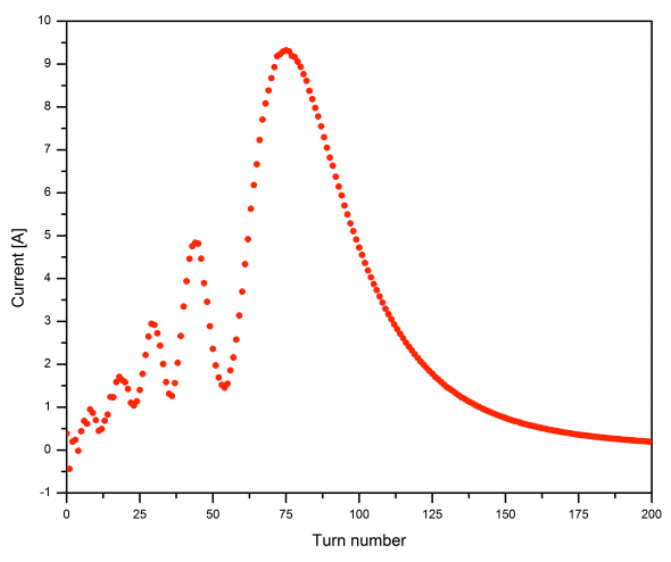

Fig 3.Current density distribution along the coil length after the optimization process (see text for details). The total length of the coil is large enough to guarantee that the current of the rings located at the extremes of the cylinder approaches zero.

In order to reproduce the optimized current density with a proper spatial distribution of rings having a unique current $I_{s}$, we sum all the contributions to get the total current $I_{T}$ and divide this value by $m$ (the desired number of discrete turns):

$$
I_{T}=\sum_{n=1}^{N} I_{n} \quad I_{s}=I_{T} / m .
$$

Here $I_{s}$ stands for the real current required from the power source. The distribution of the rings is obtained by integrating the current density curve within consecutive length intervals such that the integral value equals $I_{s}$ for all of them. In this way, we section the coil into intervals of different lengths, each representing the same integrated current. Later, the rings are placed within these intervals (one per each), at the position where the integrated current density equals at both sides of it (see figure 4).

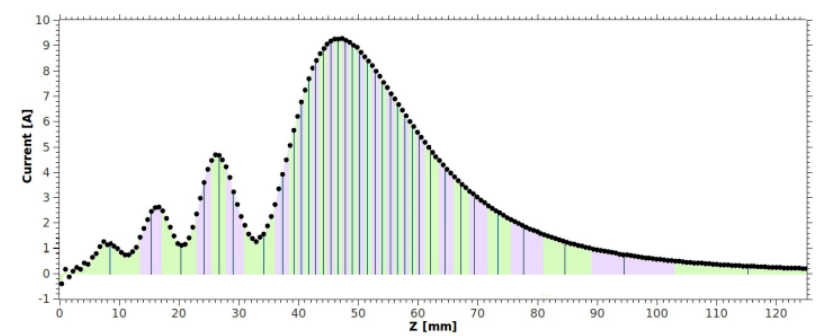

Fig 4. The figure shows the discretization process to transform $\mathrm{N}$ independent currents in a limited set of rings having all of them the same current. Solid lines show the final position of the real rings of wire (nonpitched turns). The colored vertical bands indicate intervals of identical integrated current.

The quality of the coil is calculated from the deviation of the gradient from its mean value $G_{a v}$ within the VOI [13], as well as the nonuniformity $\left(N_{u}\right)$ and nonlinearity $\left(N_{l}\right)$ [14]: 


$$
\begin{gathered}
\Delta=\frac{\left|G_{z}\left(\vec{r}_{k}\right)-G_{a v}\right|}{\left|G_{a v}\right|} \times 100 \%, \\
\text { Nonuniformity }(r)=\frac{G(r, 0)-G_{a v}}{G_{a v}} \times 100 \%, \\
\operatorname{Nonlinearity}(z)=\frac{G(0, z)-G_{a v}}{G_{a v}} \times 100 \%,
\end{gathered}
$$

\section{B. Calculation of the magnetic field gradient uniformity}

Computer calculations were performed in a home-made $\mathrm{C}++$ program. We studied the influence of $m$ on the gradient uniformity within the VOI. The radial extension of the VOI was considered with special care, since the diameter of our coil is restricted by the application constraints. Figure 5 shows that the radial extension of the uniformity zone within the VOI is roughly directly proportional to the number of discrete elements (up to $\sim 40$ ), while the driving current exhibits an inverse proportionality to this quantity (at fixed gradient intensity). This advantageous situation was appropriately exploited in our design. Longitudinal uniformity was also analyzed (not shown). A favorable compromise between the involved variables returned 35 turns as the best option (half coil, 70 turns in total).

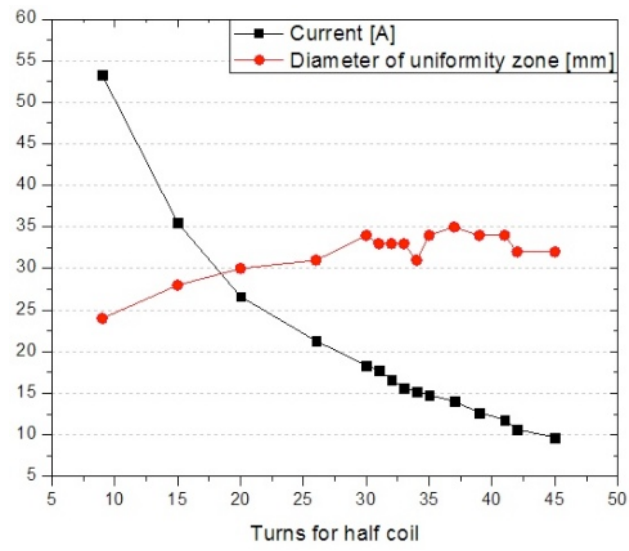

Fig 5. Driving current and radial uniformity as a function of $m$ for a fixed gradient intensity of about $200 \mathrm{mT} / \mathrm{m}$.

Figure 6 shows the calculated magnetic field gradient uniformity map corresponding to our 70-turns gradient coil. The map was obtained from a $200 \times 200$ matrix by spatial derivative of the calculated magnetic field. The green area represents a deviation of $5 \%$ in the gradient linearity (respect to a mean gradient value of $204,17 \mathrm{mT} / \mathrm{m}$, see eqn.(4)). A driving current of $15 \mathrm{~A}$ was used, which gives a calculated efficiency of $13,61 \mathrm{mT} / \mathrm{m} \bullet A$.

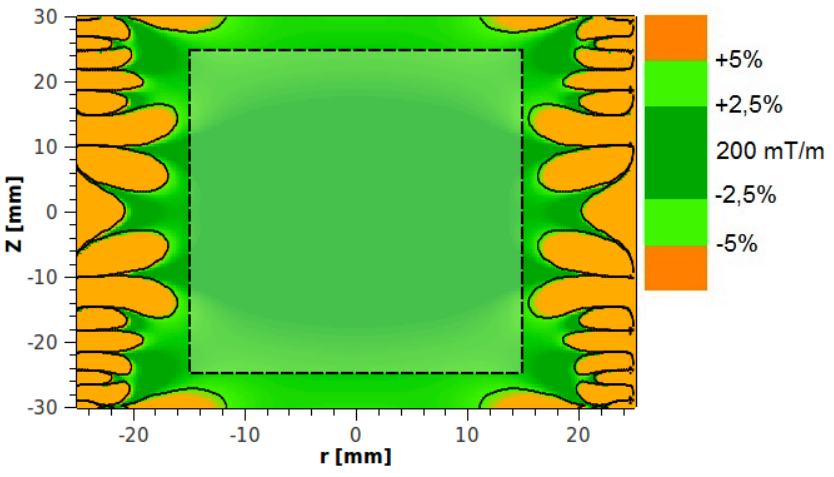

Fig 6. Uniformity map calculated for a magnetic field gradient of about $200 \mathrm{mT} / \mathrm{m}$ and a 70 -turns gradient coil. The dotted rectangle represents the sample size in our application.

\section{EXPERIMENTAL}

\section{A. Prototyping and testing set-up.}

A prototype-coil was assembled using copper wire of $0,9 \mathrm{~mm}$ in diameter ( $1 \mathrm{~mm}$ with insulation coating), wound on the outer surface of a cylindrical body machined from polyacetal resin (Delrin, Dupont). The cylinder had an external diameter of $51 \mathrm{~mm}$, an internal diameter of $47 \mathrm{~mm}$ and a total length of $231 \mathrm{~mm}$.

Magnetic field measurements were carried out using a Lake Shore 475 DSP gaussmeter with temperature compensation. The probe of the instrument was mounted on a home-made computer controlled rail allowing controlling its position with an accuracy of $(250 \pm 2) \mu \mathrm{m}$. A cylindrical insert having a diameter of $47 \mathrm{~mm}$ was machined from wood in order to guarantee the radial position of the probe during the measurements. 7 circular guides of $3 \mathrm{~mm}$ in radius and a length of $60 \mathrm{~mm}$ were milled in the wooden-insert at selected radial positions in order to slide on them the gaussmeter probe. Finally, the probe is positioned along the guides to map the magnetic field within the inner volume of the coil (figure 7).
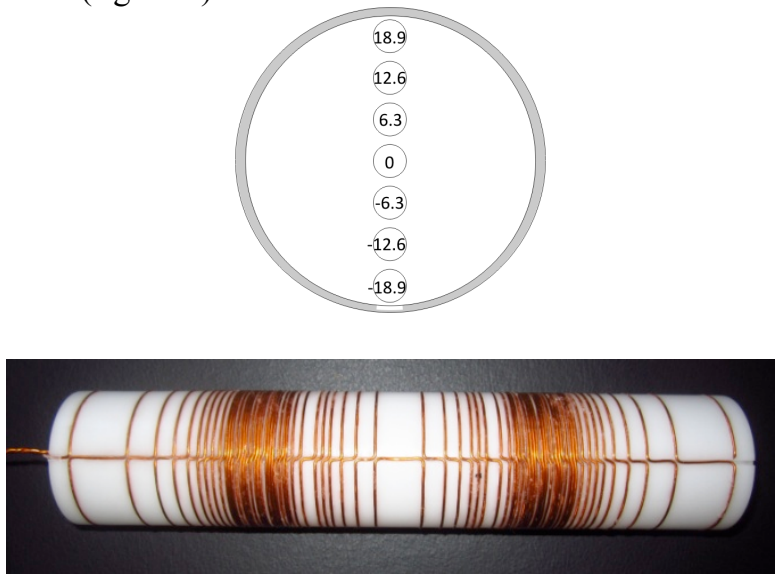

Fig 7. Positions of the milled-guides in a cross-section of the insert (units are in $\mathrm{mm}$ ). Bottom: Picture of the prototype-coil. 


\section{B. Measurements and results}

The magnetic field was measured for a current of $(4,5 \pm 0,2) \mathrm{A}$ at 23 equidistant positions separated $2,5 \mathrm{~mm}$ each other (10 steps of the step-motor), covering a longitudinal range (z-axis) from $(-27,50 \pm 0,25) \mathrm{mm}$ to $(27,50 \pm 0,25) \mathrm{mm}$ (figure 8$)$. The magnetic field was measured five times at each position. The averaged values were usedto derive the corresponding field gradient (figure 8).

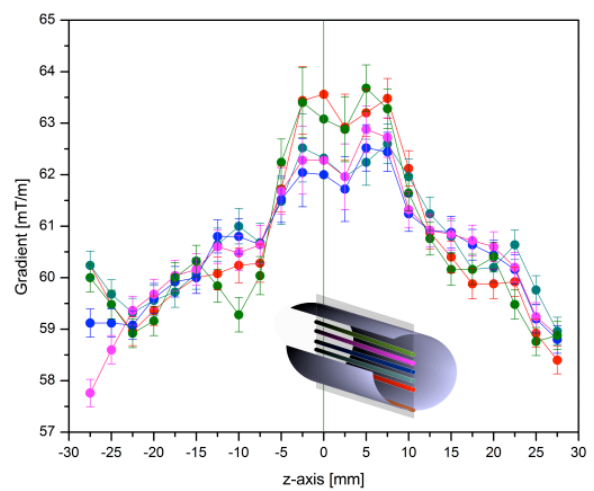

Fig 8. Magnetic field gradient along the coil (z-axis) for different radial positions. The insert shows the different radial positions as their identification with different colors. Note that even the curve corresponding to the closest position to the current return-path (most unfavorable case, brown) coincides with the rest of the radial positions. It can also be observed that the measured gradient along the z-axis within the sample size $( \pm 25 \mathrm{~mm})$ has a maximum deviation from the average value $((61 \pm 3) \mathrm{mT} / \mathrm{m})$ limited to $\pm 5 \%$. This shows the excellent performance of the designed coil.

Table 1 summarizes the main characteristics of the prototype-coil. Figure 9 represents the uniformity map obtained from the measurements.

TABLE I

CHARACTERISTIC PARAMETERS OF THE COIL

\begin{tabular}{|l|l|}
\hline \multicolumn{1}{|c|}{ Property } & \multicolumn{1}{c|}{ Value } \\
\hline Resistance $[\mathrm{m} \Omega]$ & $879 \pm 2$ \\
\hline Inductance $[\mu \mathrm{H}]$ & $122 \pm 2$ \\
\hline Efficiency $[\mathrm{mT} / \mathrm{m} \bullet \mathrm{A}]$ & $13,6 \pm 0,7$ \\
\hline$N_{u}{ }^{*}[\%]$ & $2,9 \pm 0,4$ \\
\hline$N_{l}^{*}[\%]$ & $1,8 \pm 0,3$ \\
\hline \multicolumn{2}{|c}{$* R M S$ value. }
\end{tabular}

Measurements were taken diametrically from the closest position of the current return-path to the other end. This was done to evaluate the influence of the current return-path on the gradient. No appreciable effects could be observed within experimental errors. Discrepancies between the maps of figures 6 and 9 are attributed to mechanical imprecision of the assembly and experimental errors.

The resulting radial uniformity is better than $60 \%$. The employed optimization process can also be applied for the design of transversal gradient coils by replacing the closed rings by open arcs with longitudinal return-paths.

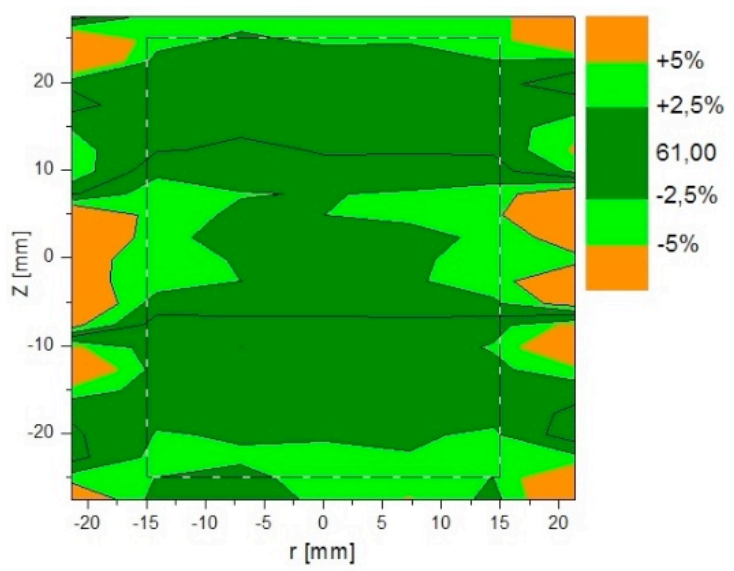

Fig 9. Measured uniformity map. The mean gradient value was measured to be $(61 \pm 3) \mathrm{mT} / \mathrm{m}$ with a driving current of $(4,5 \pm 0,2) \mathrm{A}$. The map shows a maximum deviation within the VOI that is limited to $\pm 5 \%$.

\section{CONCLUSION}

The optimization process here discussed was validated through measurements performed in a prototype-coil. The optimization, as all the approximations, has the basic weakness that a loss of accuracy occurs in the discretization of the quasi-continuous current density. Although such a loss of accuracy was not quantified in the present work, the calculations showed to be useful for the design of a gradient-coil whose performance satisfies the requirements demanded by the application. However, it is not unreasonable to hope that a refinement in the mathematical machinery, together with a more precise mechanical assembly, may allow the production of a gradient-coil with improved performance. This point will spur more theoretical and experimental efforts.

Since an optimization should be planned in view of specific purposes, a straightforward comparison with other designs may be tricky. Nevertheless, we can compare with another (experimentally tested) case where a similar cost function was used, even while having different design requirements [12]. This case fulfils a gradient uniformity (within $5 \%$ ) for a $35 \%$ of the radius. The fact that this coil performance is better in the longitudinal dimension of the field-gradient lacks relevance in our case, since in our application there are no significant technical constraints in this point. For instance, if we recalculate our coil to fit with better longitudinal uniformity, the radial performance will be degraded. Other parameters that depend on the coil dimensions will not be compared here. Another calculation using almost the same cost function was presented by Poole et. al. [15]. In this case, a better performance is shown concerning the radial uniformity, although the mathematical machinery is much more complex. However, this work only shows a theoretical model. Other designs and calculation methods can be found in the literature showing longitudinal gradient coils with similar or even better performance. However, they are in all cases computational simulations without experimental validation. 


\section{ACKNOWLEDGMENT}

This work was supported by Foncyt PICT2008-1810, CONICET PIP2010-2012 11220090100663 and Secyt-UNC.

\section{REFERENCES}

[1] P. Mansfield and P. K. Grannell, "NMR 'diffraction' in solids?", $J$. Phys. C: SolidState Phys., vol. 6, p. 422, Nov. 1973.

[2] P. C. Lauterbur, "Image formation by induced local interactions: Examples Employing Nuclear Magnetic Resonance", Nature, vol. 242, p. 190, Mar. 1973.

[3] E. Anoardo, G. Galli and G. Ferrante, "Fast-field-cycling NMR: Applications and instrumentation", Appl. Mag. Reson., vol. 20, p. 365, April 2001

[4] R. Kimmich and E. Anoardo, "Filed-cycling NMR relaxometry", Prog. Nucl. Mag. Reson. Spectrosc., vol. 44, p. 257, Feb. 2004.

[5] G. Ferrante and S. Sykora, "Technical aspects of the field cycling", Adv. Inorg. Chem., vol. 57, p. 405, April 2005.

[6] Abragam and W. G. Proctor, "Spin temperature",Phys. Rev.,vol. 109, p. 1441, Mar. 1958.

[7] S. H. Koenig and R. D. III Brown, "Magnetic field dependence of 1/T1 of protons in tissue“, Invest. Radiol., vol. 19, p. 76, Mar. 1984.

[8] J. W. Carlson, D. M. Goldhaber, A. Brito and L. Kaufman, "MR relaxometry imaging. Work in progress.”,Radiology,vol .184, p. 635, Sep. 1992.
[9] N. I. Matter, G. C. Scott, T. Grafendorfer, A. Macovski and S. M. Conolly, „Rapid Polarizing Field Cycling in Magnetic Resonance Imaging“", IEEE Trans. Med. Imag., vol. 25, p.84, January 2006.

[10] D. J. Lurie, S. Aime, S. Baroni, N. A. Booth, L. M. Broche, C.-H Choi, G. R. Davies, S. Ismail, D. Ó hÓgáin and K. J. Pine, "Fast field-cycling magnetic resonance imaging", C. R. Physique, vol. 11, p.136, July 2010 .

[11] S. Kruber, G. D. Farrher and E. Anoardo, "New magnet design for fast-field-cycling Nuclear Magnetic Resonance",IEEE Lat. Am. Trans.,vol. 11, p. 252, Feb. 2013.

[12] R. Lemdiasov and R. Ludwing, "A stream function method for gradient coil design”,ConceptsMagn. Reson. B,vol .26, p. 67, Mar. 2005.

[13] E. C. Wong, A. Jesmanowicz and J. S. Hyde, "Coil optimization for MRI by conjugate gradient descent", Mag. Reson. Med., vol. 21, p. 39, September 1991.

[14] Sh. Shvartsman, R. Brown, Y. Cheng, T. Eagan, H. Fujita, M. Morich, L. Petropoulos and J. Willig, "A new 'supershilding' method applied to the design of gradient coils", Magn. Reson. Med., vol. 45, p. 147, January 2001.

[15] M. Poole, R. Bowtell, "Novel gradient coils using a boundary element method", Concepts Magn. Reson. B, vol. 31, p. 162, August 2007. 